\title{
Intact Cell Lipidomics Reveal Changes to the Ratio of Cardiolipins to Phosphatidylinositols in Response to Kanamycin in HeLa and Primary Cells
}

\author{
Sonia Rebollo-Ramirez, ${ }^{\dagger}$ Sina Krokowski, ${ }^{\ddagger}, \S$ Damian Lobato-Márquez, ${ }^{\ddagger} \S$ Michael Thomson, ${ }^{\dagger}$ \\ Ivana Pennisi, ${ }^{\dagger}$ Serge Mostowy, ${ }^{\ddagger}$, and Gerald Larrouy-Maumus*, ${ }^{, \dagger}$
}

${ }^{\dagger}$ MRC Centre for Molecular Bacteriology and Infection, Department of Life Sciences, Faculty of Natural Sciences, Imperial College London, London SW7 2AZ, U.K.

${ }^{\ddagger}$ MRC Centre for Molecular Bacteriology and Infection, Department of Medicine, Section of Microbiology, Imperial College London, London W12 0NN, U.K.

${ }^{\S}$ Department of Immunology and Infection, London School of Hygiene and Tropical Medicine, Keppel Street, London WC1E 7HT, U.K.

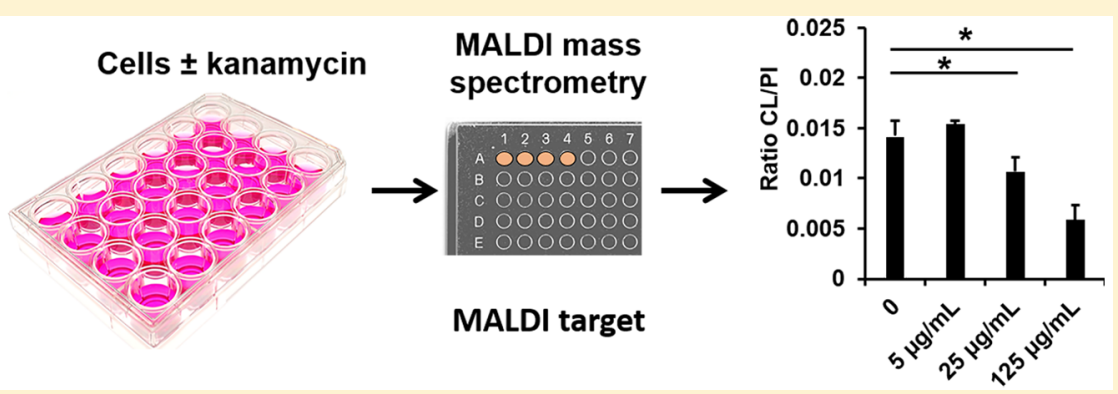

ABSTRACT: Antimicrobial resistance is a major threat the world is currently facing. Development of new antibiotics and the assessment of their toxicity represent important challenges. Current methods for addressing antibiotic toxicity rely on measuring mitochondrial damage using ATP and/or membrane potential as a readout. In this study, we propose an alternative readout looking at changes in the lipidome on intact and unprocessed cells by matrix-assisted laser desorption ionization mass spectrometry. As a proof of principle, we evaluated the impact of known antibiotics (levofloxacin, ethambutol, and kanamycin) on the lipidome of HeLa cells and mouse bone marrow-derived macrophages. Our methodology revealed that clinically relevant concentrations of kanamycin alter the ratio of cardiolipins to phosphatidylinositols. Unexpectedly, only kanamycin had this effect even though all antibiotics used in this study led to a decrease in the maximal mitochondrial respiratory capacity. Altogether, we report that intact cell-targeted lipidomics can be used as a qualitative method to rapidly assess the toxicity of aminoglycosides in HeLa and primary cells. Moreover, these results demonstrate there is no direct correlation between the ratio of cardiolipins to phosphatidylinositols and the maximal mitochondrial respiratory capacity.

\section{INTRODUCTION}

Given their potential side effects, antibiotics can be a doubleedged sword. For instance, aminoglycosides can cause hearing loss as well as kidney damage in humans. ${ }^{1,2}$ Several lines of evidence have demonstrated that clinically relevant doses of antibiotics induce the formation of reactive oxygen species (ROS) and mitochondrial dysfunction in mammalian cells, due to disruption of the tricarboxylic acid (TCA) cycle and the electron transport chain (ETC). ${ }^{3-7}$ Thus, assessment of antibiotic toxicity is a crucial factor to address in drug discovery. For example, troglitazone, ${ }^{8}$ an antidiabetic and anti-inflammatory drug, and cerivastatin, ${ }^{9}$ a member of the class of cholesterollowering drugs, were withdrawn from the market in the early 2000s because of their toxicity to mitochondrial function. Importantly, between 1994 and 2006, 38 antibiotics approved by the U.S. Food and Drug Administration were withdrawn, representing $\sim 2 \%$ of the total drugs commercially available. ${ }^{10,11}$ Therefore, there is an urgent need to not only develop better antibiotics but also to select antibiotics that do not generate ROS, mitochondrial damage, or other negative side effects. Currently, a variety of commercially available assays are available to measure the effect of antibiotic toxicity in mitochondria, based on measurements of ATP levels or changes in membrane potential. Moreover, other technologies can assess antibiotic toxicity by measuring mitochondrial oxygen consumption using oxygen sensors and time-resolved fluorescence. However, these solutions can be time-consuming and expensive. In this study,

Received: February 10, 2018

Published: June 27, 2018 
we propose a new method for assessing antibiotic toxicity based on intact cell lipid profiling. Antibiotics can alter the central carbon metabolism and therefore the TCA cycle and the ETC, which consequently leads to a decrease in metabolic activity and changes in metabolic pathways. ${ }^{12,13}$ Among these metabolic pathways, we reasoned that fatty acid synthesis can be altered as a result of a changes in the TCA cycle activity, and as a consequence an alteration of available levels of acetyl-coenzyme A required for lipids synthesis. We therefore propose that changes in the TCA cycle activity could lead to a remodeling of the cell lipidome, and these changes can be used as potential markers of antibiotic toxicity. The cell lipidome includes lipids such as phospholipids (PLs), phosphatidylinositols (PI), and cardiolipins (CL). CL or diphosphatidylglycerols are found almost exclusively in the inner membrane of the mitochondria and are associated with enzymes and oxidative phosphorylation complexes involved in ATP biosynthesis and the maintenance of the ETC. ${ }^{14,15}$ We thus hypothesize that lipidomics and highthroughput technologies can be used as an alternative to probe changes in the relative abundance of PI and CL as a readout of mitochondrial damage resulting from antibiotic toxicity. To have access to the entire lipidome and because of the heterogeneity of the lipids, extraction procedures (which enrich lipids and prefractionate them) can be crucial for evaluating the changes in the lipidome. ${ }^{16-20}$ The conventional separation of lipid classes is predominantly achieved by differential solvent extraction, followed by silica thin-layer chromatography, gas chromatography, or liquid chromatography such as normalphase or hydrophobic interaction liquid chromatography (HILIC). ${ }^{21-23}$ Over the past decade, the capabilities of matrix-assisted laser desorption ionization mass spectrometry (MALDI-MS) in lipid analysis have been demonstrated for the analysis of lipid extracts from different biological materials. ${ }^{24-28}$ However, the most promising advantage of the MALDI-MS technique is performing lipid analysis avoiding extraction and/ or separation steps, called intact cell lipidomics (ICL). ICL is highly valuable for lipids that are tightly bound to membrane proteins (e.g., CL) and may be difficult to completely recover in lipid extracts. For example, Angelini and colleagues reported the analysis of lipidomics of yeast (Saccharomyces cerevisae) and bovine heart mitochondria by first isolating mitochondria followed by direct MALDI-MS using the matrix 9-amino acridine solubilized in the organic solvents 2-propanol and acetonitrile in a $6: 4(\mathrm{v} / \mathrm{v})$ ratio. $^{24}$ In this case, they could identify in the negative ion mode phosphatidic acid (PA), phosphatidylserine (PS), phosphatidylglycerol (PG), phosphatidylethanolamine (PE), PI, and CL. As another example, Jones and colleagues performed ICL on yeast by MALDI-Fourier transform-MS in untreated yeast using a saturated solution of the matrix 2,5-dihydroxybenzoic acid dissolved in a 9:1 ( v/v) methanol/water mixture, allowing the detection in the positive ion mode of phosphatidylcholine (PC), PI, PE, and PA. ${ }^{29}$ Although highly promising, the study by Angelini and colleagues relies on isolation of organelles (such as the mitochondria or membranes), which is incompatible with high-throughput screening (HTS) for assessing antibiotic toxicity and mitochondrial damage. Furthermore, the study by Jones and colleagues includes several steps of sample preparation prior to ICL. As such, several washes with the appropriate buffer followed by suspension of the pellet in 50\% methanol are required prior to ICL, and the solution must be deposited within $10 \mathrm{~min}$ on the target, making this sample preparation challenging for HTS. So far, studies have never investigated lipidome remodeling in response to a trigger using ICL on intact nonprocessed eukaryotic cells. We recently reported bacterial lipidomics on intact mycobacteria and Enterobacteriacae without any isolation of membranes or subcellular compartments, and without any sample preparation other than directly loading the samples on the MALDI target followed by the addition of the matrix solubilized in organic solvents. ${ }^{30,31}$ Considering this success, we sought to apply a similar approach to intact untreated and antibiotic-treated eukaryotic cells to evaluate the potential of this technology in the assessment of the effect of the antibiotic on the lipidome. In this study, we chose two cell types, a cell line and primary cells. HeLa cells, a human epithelial immortal cell line, are used as model for this study because of their widespread use for the investigation of host-pathogen interactions. HeLa cells can be invaded by a wide variety of well-studied enteropathogens, such as Salmonella, Shigella, Listeria, and Yersinia. ${ }^{32-35}$ Mouse bone marrow-derived macrophages (BMDM) are primary cells widely used to study host-pathogen interactions, too. As a proof of principle and to demonstrate the potential of ICL, we focused on determining the impact on the targeted lipidome on cells treated with the aminoglycoside, kanamycin. Kanamycin is an antibiotic that blocks protein synthesis by binding to the bacterial ribosomal subunit. ${ }^{36}$ Kanamycin is broadly used to treat severe bacterial infection worldwide but has been associated with drastic side effects. To evaluate the specificity of kanamycin's impact on the lipidome of the cells, we also used two other antibiotics with different modes of action, the fluoroquinolone levofloxacin ${ }^{37,38}$ that inhibits bacterial DNA gyrase and DNA topoisomerase IV $^{39}$ and ethambutol $^{40}$ that targets the arabinosyltranferases EMBs involved in arabinogalactan biosynthesis, which is a major constituent of the mycobacterial cell wall. ${ }^{41}$ Importantly, all antibiotics employed in this study are currently used for the treatment of bacterial infections in clinics. The concentrations used in our study were chosen according to the peak of antibiotic concentration found in the serum $\left(C_{\max }\right)$ of patients treated with these antibiotics. ${ }^{6,37,38,40,42}$ Remarkably, by using ICL on intact HeLa and BMDM cells, we found that the ratio of CL to PI is impaired upon treatment with clinically relevant concentrations of kanamycin and high levels of levofloxacin $\left(5 C_{\max }\right)$. However, by investigating mitochondrial respiration, we found, for the first time, no direct correlation between the CL:PI ratio and mitochondrial damage.

\section{MATERIALS AND METHODS}

HeLa Cell Growth Conditions. HeLa (ATCC CCL) cells were maintained in Dulbecco's minimal essential medium (DMEM, Gibco by Life Technologies) supplemented with $10 \%$ heat-inactivated fetal bovine serum at $37^{\circ} \mathrm{C}$. Cells $\left(1-5 \times 10^{5}\right.$ cells $/ \mathrm{mL}$, which is the lowest limit for performing the experiments) were seeded into 24-well tissue culture plates (Corning). Cells were allowed to adhere overnight, then scraped off, and washed three times with $500 \mu \mathrm{L}$ of doubly distilled $\mathrm{H}_{2} \mathrm{O}$ $\left(\mathrm{ddH}_{2} \mathrm{O}\right)$ by centrifuging them at $15,000 \times g$ for $5 \mathrm{~min}$ and carefully discarding the supernatant. The final pellet was resuspended in $100 \mu \mathrm{L}$ of $\mathrm{ddH}_{2} \mathrm{O}$.

Isolation of Mouse Bone Marrow-Derived Macrophages. Bone marrow cells were flushed from the femur and tibia of 8-10-weekold female BALB $/ \mathrm{c}$ mice and differentiated into macrophages for 7 days in RPMI 1640 (Gibco) supplemented with $1 \mathrm{mM}$ sodium pyruvate (Gibco), $2 \mathrm{mM}$ L-glutamine (Lonza), $0.05 \mathrm{M}$ 2-mercaptoethanol (Gibco), 10\% heat-inactivated fetal bovine serum (Biosera), and 20\% L-cell conditioned media. On day 4, cells were fed with an additional 10 $\mathrm{mL}$ of media. After being cultured for 7 days, cells were washed with phosphate-buffered saline (PBS) and seeded at a density of $5 \times 10^{5}$ 

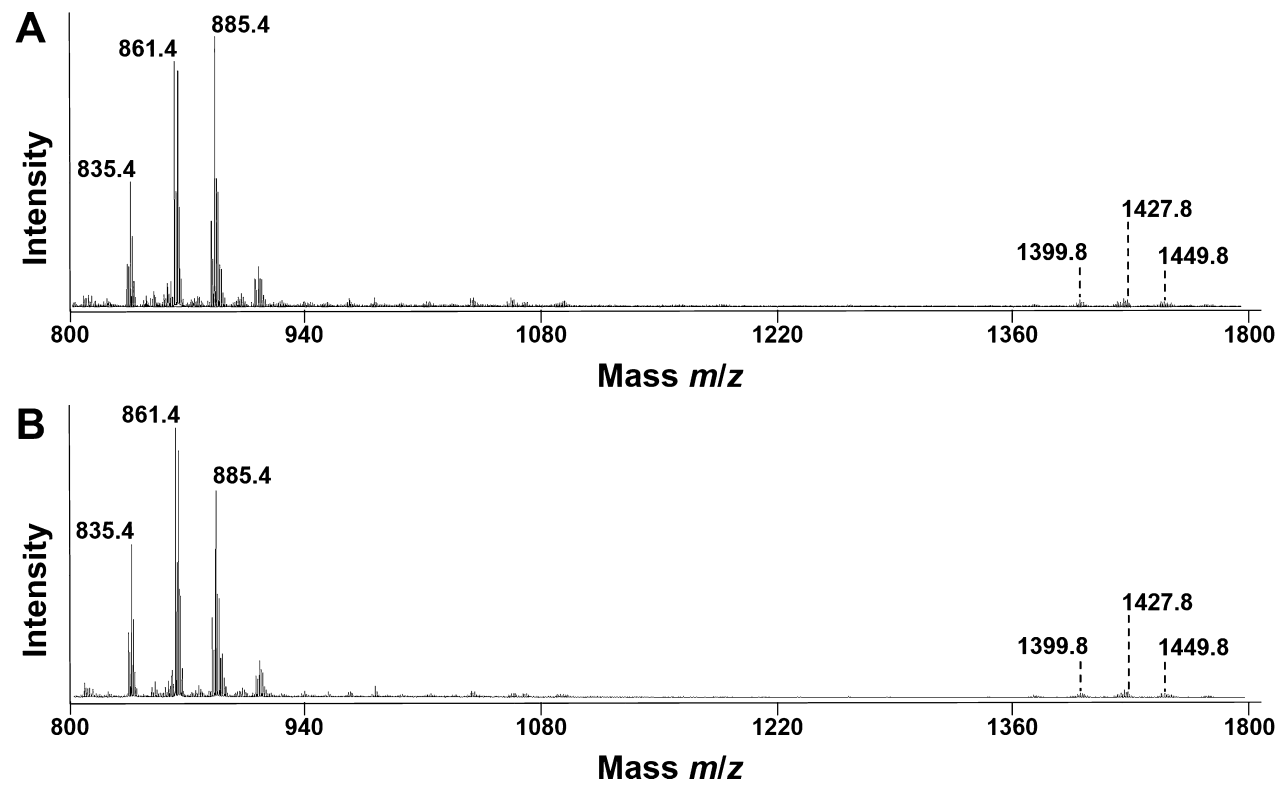

Figure 1. Lipid fingerprint of intact HeLa cells by MALDI-MS. (A) Negative ion mode MALDI-TOF MS spectra of intact untreated HeLa cells at $24 \mathrm{~h}$. (B) Negative ion mode MALDI-TOF MS spectra of intact HeLa cells treated with $25 \mu \mathrm{g} / \mathrm{mL}$ kanamycin for $24 \mathrm{~h}$. Compared to untreated HeLa cells in panel A, where the major peak is at $m / z 885.4$, in kanamycin-treated cells, the major peak is at $m / z$ 861.4, demonstrating a change in PI metabolism upon treatment with kanamycin compared to untreated HeLa cells.

cells/mL for lipidomics and the Seahorse XF cell mito stress test and 9,000 cells/well for cell viability assays.

Seahorse XF Cell Mito Stress Test. Mouse bone marrow-derived macrophages and HeLa cells were seeded on an eight-well miniplate at cell densities of $5 \times 10^{5}$ and $1.5 \times 10^{5}$ cells $/ \mathrm{mL}$, respectively, and left to adhere overnight. Cells were or were not treated with antibiotics, and after $24 \mathrm{~h}$, the oxygen consumption rate (OCR) was measured using a Seahorse XFp extracellular flux analyzer (Seahorse Bioscience). The day before the assay, the sensor cartridge was hydrated by filling the utility plate with $200 \mu \mathrm{L}$ of sterile $\mathrm{dd}_{2} \mathrm{O}$ and incubating overnight at $37^{\circ} \mathrm{C}$ in a non- $\mathrm{CO}_{2}$ incubator. On the day of the assay, the water was replaced with $200 \mu \mathrm{L}$ of prewarmed XF Calibrant (Agilent) and incubated for $1 \mathrm{~h}$ at $37^{\circ} \mathrm{C}$ in a non- $\mathrm{CO}_{2}$ incubator. Also, on the day of the assay, the culture medium was replaced with $180 \mu \mathrm{L}$ of unbuffered Seahorse XF medium [DMEM with phenol red supplemented with 10 $\mathrm{mM}$ glucose, $1 \mathrm{mM}$ sodium pyruvate, $2 \mathrm{mM}$ glutamine, and phenol red $\mathrm{pH}$ 7.4], and the cells were incubated at $37^{\circ} \mathrm{C}$ in a non- $\mathrm{CO}_{2}$ incubator for $45 \mathrm{~min}$. Baseline rates were measured at $37^{\circ} \mathrm{C}$ three times before sequentially injecting the following mitochondrial inhibitors: oligomycin $(10 \mu \mathrm{M})$, carbonyl cyanide $p$-(trifluoromethoxy) phenylhydrazone (FCCP, $20 \mu \mathrm{M})$, and a rotenone/antimycin A mixture $(5 \mu \mathrm{M})$. After the addition of each inhibitor, three readings were taken. OCR was automatically calculated by the Seahorse XFp software. Every point represents an average of three different wells.

Cell Viability Assay. Cell viability was assessed using Alamar Blue Cell Viability Reagent (catalog no. DAL1025) purchased from Thermo Fisher Scientific and by following the manufacturer's instructions; 9,000 cells per well were seeded on a black, clear bottom 96-well plate (Corning). Cells were or were not treated with antibiotics for $24 \mathrm{~h} ; 5 \%$ DMSO (Sigma) in medium was used as a positive control. The fluorescence intensity was monitored using a HIDEX microplate reader with excitation and emission wavelengths set at 544 and $590 \mathrm{~nm}$, respectively. The assay was performed in quadruplicate and repeated three times.

Antibiotic Treatments. Cells were treated for $24 \mathrm{~h}$ at $37^{\circ} \mathrm{C}$ in an incubator with $5 \% \mathrm{CO}_{2}$ with ${ }_{5}{ }_{5} C_{\max }, C_{\max }$ and $5 C_{\max }$ corresponding to $0.8,4$, and $20 \mu \mathrm{g} / \mathrm{mL}$ for ethambutol, 5, 25, and $125 \mu \mathrm{g} / \mathrm{mL}$ for kanamycin, and $2.2,11$, and $55 \mu \mathrm{g} / \mathrm{mL}$ for levofloxacin, respectively. After being exposed for $24 \mathrm{~h}$, cells were then used for further analysis.

MALDI-MS Analysis. Prior to mass spectrometry analysis, the super-2,5-dihydroxybenzoic acid (Sigma-Aldrich, catalog no. 50862) matrix was added at a final concentration of $10 \mathrm{mg} / \mathrm{mL}$ in a chloroform/ methanol mixture at a 90:10 (v/v) ratio; $0.4 \mu \mathrm{L}$ of a cell solution at a concentration of $2 \times 10^{5}$ to $2 \times 10^{6} \mathrm{~mL}^{-1}$, corresponding to $\sim 100-$ 1000 cells per well of the MALDI target plate (384 Opti-TOF $123 \mathrm{~mm}$ $\times 84 \mathrm{~mm} \mathrm{AB}$ Sciex NC0318050, 1016629), and $0.6 \mu \mathrm{L}$ of the matrix solution were deposited on the MALDI target plate, mixed with a micropipette, and left to dry gently. MALDI-TOF MS analysis were performed on a 4800 Proteomics Analyzer (with TOF-TOF Optics, Applied Biosystems) using the reflectron mode. Samples were analyzed operating at $20 \mathrm{kV}$ in the negative ion mode, and three independent experiments were performed. Mass spectrometry data were analyzed using Data Explorer version 4.9 from Applied Biosystems. Assignments were based on the MS/MS fragmentation profile and CL standards from bovine heart (Sigma-Aldrich, catalog no. C0563).

Membrane Potential Measurement. 6,000 HeLa cells per well were seeded on a black sterile 96 -well plate with a clear flat bottom. After $24 \mathrm{~h}$, cells were treated with $25 \mu \mathrm{g} / \mathrm{mL}$ kanamycin for $24 \mathrm{~h}$ and changes in membrane potential were measured by fluorescence with excitation at $475 \mathrm{~nm}$ and emission at $530 \mathrm{~nm}$ following the protocol of the JC-1-Mitochondrial Membrane potential assay kit and as recommended by the manufacturer (ab113850).

Preparation of Microscopy Samples. HeLa cells were plated (1$5 \times 10^{5}$ ) on glass coverslips in six-well plates (Thermo Scientific) and used for experiments $24 \mathrm{~h}$ later. HeLa cells were treated with $25 \mu \mathrm{g} / \mathrm{mL}$ kanamycin for $24 \mathrm{~h}$ and incubated with $0.1 \mu \mathrm{M}$ MitoTrackerTM Red CMXRos for $30 \mathrm{~min}$ prior to fixation. HeLa cells were washed with $1 \times$ PBS and fixed in 4\% paraformaldehyde (PFA) for $15 \mathrm{~min}$ at room temperature. Then, cells were washed with $1 \times$ PBS, incubated for 10 min in $1 \mu \mathrm{g} / \mathrm{mL}$ DAPI, and mounted in aqua polymount mounting medium (Polyscience Inc.).

Microscopy. Samples were imaged on an Axiovert Z1 instrument driven by ZEN software (Carl Zeiss MicroImaging) taking Z-stack image series using a $63 \times$ oil objective. Image deconvolution was performed using ZEN software, and mitochondrial morphology was analyzed using the MiNA-master plugin for FIJI. At least 100 cells per experiment from three independent experiments were used for analysis. Image processing was performed in Icy (http://icy.bioimageanalysis. org).

Statistical Analysis. Data are presented as means \pm the standard error of the mean from at least three independent experiments and three technical replicates per condition for power calculation. Student's 
$t$ tests were used to compare values, with $p<0.05$ being considered significant. Ratios of changes (e.g., CL:PI ratio, cell viability, and membrane potential) were determined compared to untreated cells, and the standard deviation was calculated according to the error propagation in calculated ratios. ${ }^{43}$

\section{RESULTS AND DISCUSSION}

ICL Identified Changes in the CL:PI Ratio in Kanamycin-Treated HeLa Cells. To detect PIs and CLs (which are negatively charged molecules) by ICL, the mass spectra of lipids of intact HeLa cells in the absence or presence of kanamycin were acquired in the negative ion mode using super2,5-dihydroxybenzoc acid as the matrix (Figure $1 \mathrm{~A}, \mathrm{~B}$ ). This matrix was chosen because of its versatility for the analysis of phospholipids. ${ }^{44}$

In the negative ion mode, for both untreated and kanamycintreated $(25 \mu \mathrm{g} / \mathrm{mL}$ for $24 \mathrm{~h})$ HeLa cells, all signals in the mass spectrum can be attributed to the $[\mathrm{M}-\mathrm{H}]^{-}$molecular ions and fall into two main categories. The peaks can be grouped into two main $m / z$ ranges: (i) $m / z 800-950$, attributable to major PIs, and (ii) $m / z 1300-1500$, attributable to CLs. In the range of $m /$ $z$ 700-950, consisting of major PIs, the peaks at $m / z$ 835.4, $861.4,863.4,885.4$, and 911.5 are assigned to PI $34: 1,36: 2,36: 1$, $38: 4$, and $40: 4$, respectively, and supported by their MS/MS profile (Figure.S1). In the CL range, the major peaks at $\mathrm{m} / \mathrm{z}$ 1399.8 and 1427.8 can be attributed to CL 68:4 and 70:4 where the major species are $\mathrm{CL}(16: 1 / \mathrm{C} 18: 1)_{2}$ and $\mathrm{CL}(16: 1 / \mathrm{C} 18: 1 /$ 18:1/18:1), respectively, and are supported by their MS/MS profile (Figures S2 and S3). These data are consistent with results from published lipidome analysis ${ }^{24,29}$ but here are generated on intact and unprocessed biological samples. However, as compared to untreated cells, kanamycin-treated cells significantly changed the relative abundance of main PI lipids, with an increase in PI lipids containing shorter fatty acid chain lengths at $m / z 835.4,861.4$, and 863.4 compared to $m / z$ 885.4 , as an internal reference (Figure 1B). By using the ratio of the sum of the abundance of PI 34:1,36:2, and 36:1 to PI 38:4, a $25 \%$ increase in this ratio can be observed in the presence of kanamycin, compared to that in untreated cells (Figure 2A). Furthermore, a $40 \%$ decrease in the CL:PI ratio is observed in kanamycin-treated cells at $24 \mathrm{~h}$, as compared to that in untreated cells (Figure 2B). Taken together, these data support an alteration in PI metabolism in response to kanamycin treatment.

In summary, these data show that kanamycin treatment results in the production of PI containing shorter fatty acids and alteration of the CL:PI ratio. These results suggest an impairment of the bioenergetics of mitochondria caused by a decrease in the TCA cycle activity and fatty acid synthesis.

Kanamycin Alters the Membrane Potential and Mitochondrial Morphology in HeLa Cells. PLs (PI and $\mathrm{CL}$ ) are associated with membranes that can generate an electrochemical gradient involved in mitochondrial bioenergetic processes. Considering that mitochondrial energy metabolism is tightly linked to organelle function, disruption of the ETC should lead to a decrease in the mitochondrial membrane potential, ATP levels, and overall metabolic activity. ${ }^{45,46}$ Membrane potential measurements provide information about the coupling of electron transfer to ATP synthesis, as well as the organelle's ability to take up and release ions and substrates across the mitochondrial inner membrane. We therefore performed mitochondrial membrane potential measurements to confirm that the observed lipid changes are due to disrupted
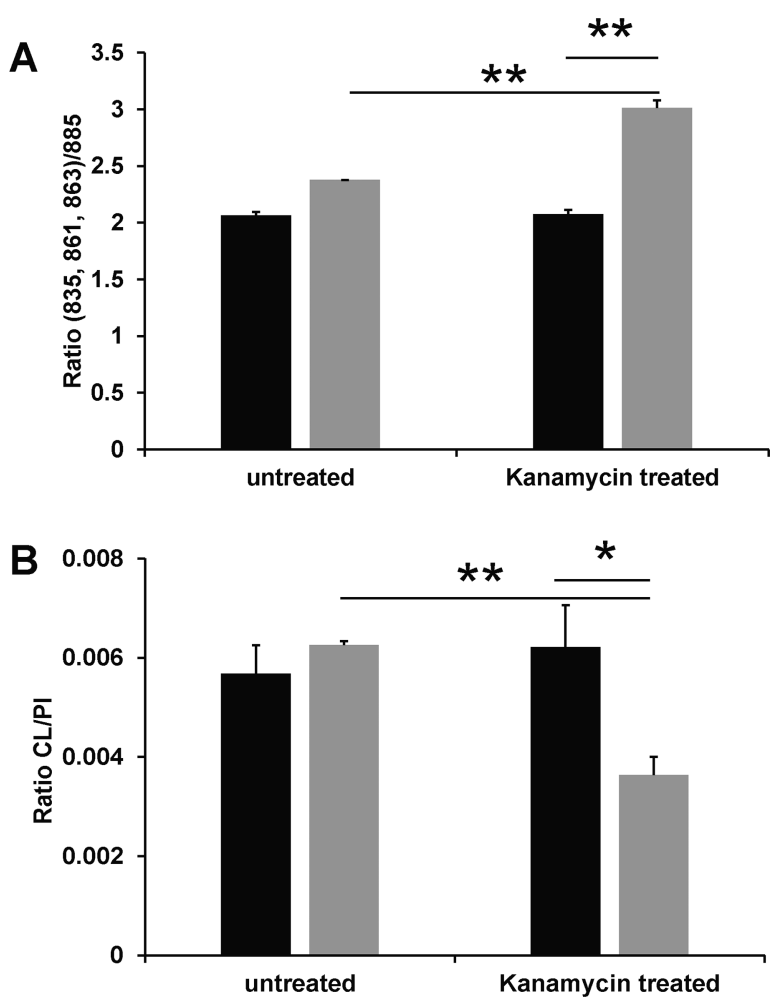

Figure 2. Kanamycin at $25 \mu \mathrm{g} / \mathrm{mL}$ for $24 \mathrm{~h}$ induces changes in phospholipids in HeLa cells. (A) Ratio of the sum of the abundance of PI (34:1, 36:2, and 36:1) to PI 38:4. (B) Ratio of CL to PI lipids. Black bars represent time zero, and gray bars represent $24 \mathrm{~h} . * p<0.05$ and $* * p<0.005$, as analyzed by a Student's $t$ test.

mitochondrial bioenergetics and not an indirect effect of our experimental design.

HeLa cells were treated for $24 \mathrm{~h}$ with $25 \mu \mathrm{g} / \mathrm{mL}$ kanamycin, and the mitochondrial membrane potential was measured and expressed as a ratio compared to untreated cells (Figure 3). In

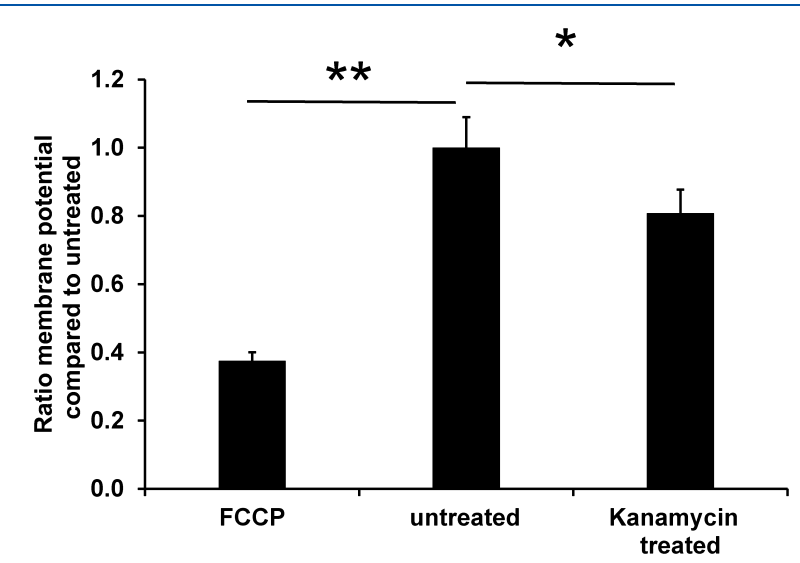

Figure 3. Mitochondrial membrane potential of HeLa cells after treatment for $24 \mathrm{~h}$ with $25 \mu \mathrm{g} / \mathrm{mL}$ kanamycin. ${ }^{*} p<0.05$ and $*^{*} p<$ 0.005 , as analyzed by a Student's $t$ test.

this case, we observed a $20 \%$ decrease in the membrane potential compared to that of untreated cells, which is consistent with observations from the literature. ${ }^{6}$ Comparison of the membrane potential of a control with that of FCCP [carbonyl cyanide 4(trifluoromethoxy) phenylhydrazone], an ionophore uncoupler of oxidative phosphorylation, showed it decreased by $60 \%$ 

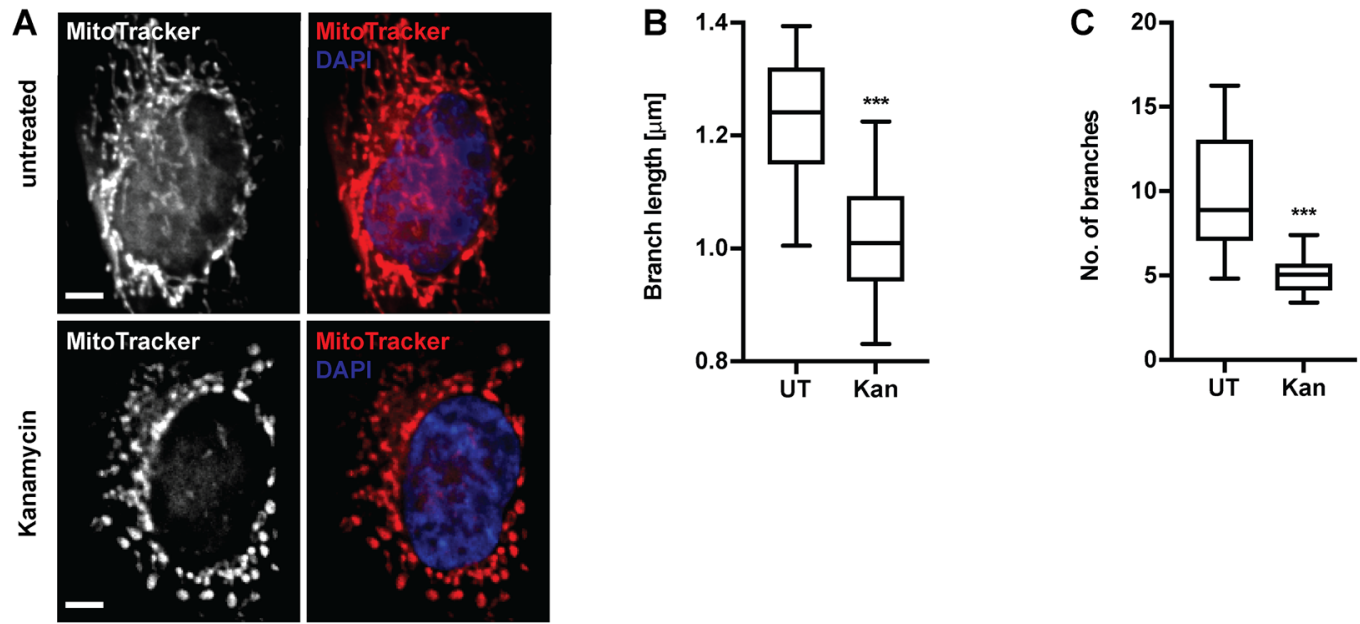

Figure 4. Mitochondrial morphology in untreated and kanamycin-treated HeLa cells. (A) HeLa cells were left untreated (UT) and treated with kanamycin for $24 \mathrm{~h}$ (Kan). Mitochondrial morphology was analyzed in HeLa cells using MitoTracker Red CMXros. The scale bar represents $5 \mu \mathrm{m}$. (B) Mitochondrial branch length measured using the MiNA-master plugin for FIJI. This graph shows median and whiskers (minimum to maximum). (C) Number of mitochondrial branches determined using the MiNA-master plugin for FIJI. This graph shows median and whiskers (minimum to maximum). $* * * p<0.001$, as analyzed by a Student's $t$ test.
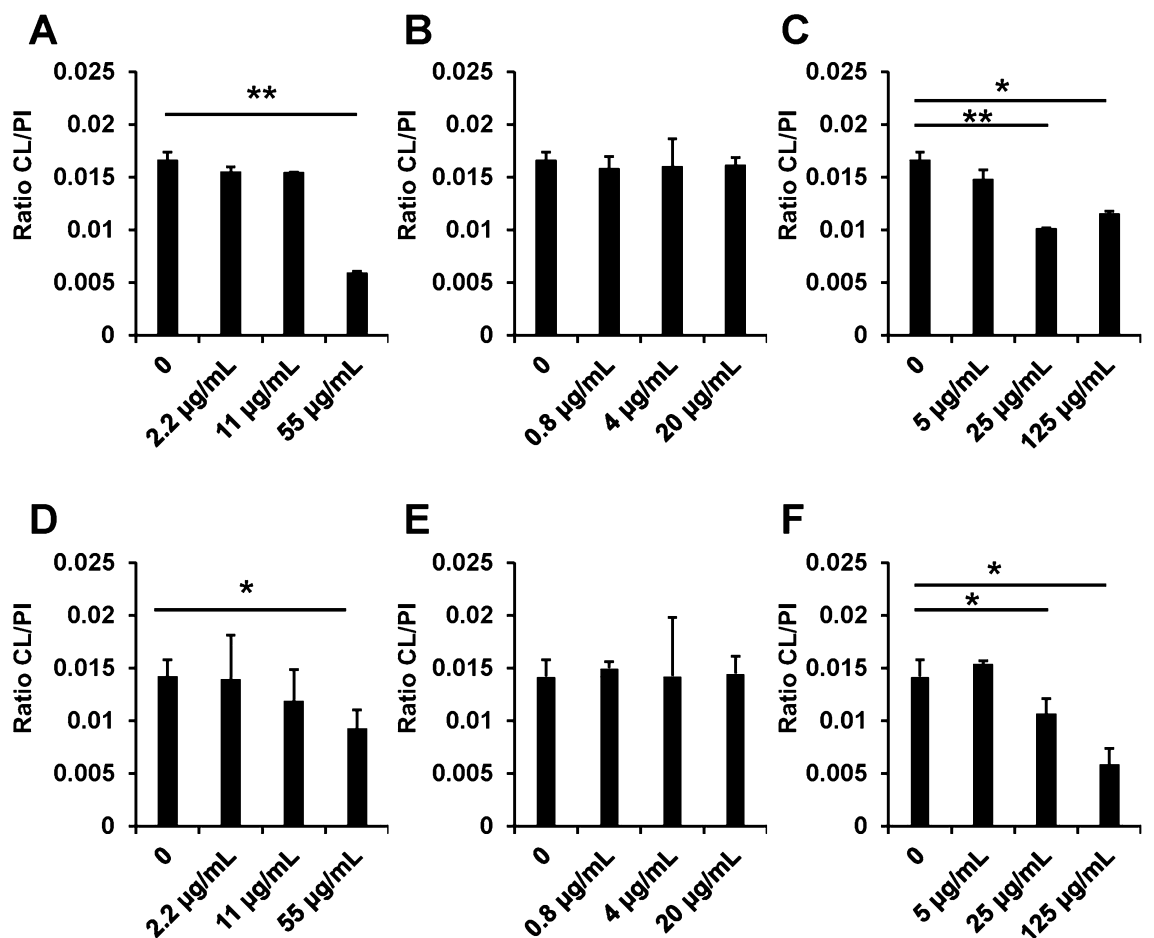

Figure 5. CL:PI ratios at ${ }^{1} /{ }_{5} C_{\max } C_{\max }$ and $5 C_{\max }$ for HeLa cells and BMDM cells treated with levofloxacin (A and D, respectively), ethambutol (B and $\mathrm{E}$, respectively), and kanamycin (C and F, respectively). ${ }^{*} p<0.05$ and ${ }^{*} p<0.005$, as analyzed by a Student's $t$ test.

compared to that of untreated cells. To further validate our methodology, we investigated possible changes in mitochondrial morphology after kanamycin treatment using microscopy to determine the number of mitochondrial branches and their length as a readout of changes in mitochondrial bioenergetics. Effectively, more and longer branches presented by mitochondria may represent a larger surface area, suggesting a higher level of potential energy to be spent. Smaller mitochondria and mitochondria with simpler shapes would suggest a decrease in their energy level. ${ }^{47}$ To investigate this, HeLa cells were or were not treated with kanamycin $(25 \mu \mathrm{g} / \mathrm{mL}$ for $24 \mathrm{~h})$. Untreated cells showed longer and highly branched mitochondria, whereas cells treated with kanamycin presented shorter and less branched mitochondria (Figure 4). In particular, mitochondrial branch lengths were significantly shorter and the number of branches was smaller in kanamycin-treated HeLa cells than in the untreated ones (Figure 4B,C).

Collectively, these data show that a clinically relevant concentration of kanamycin alters mitochondrial morphology and membrane potential.

ICL Probed Whether Kanamycin but Not Levofloxacin or Ethambutol Induces Changes in the CL:PI Ratio in a Dose-Dependent Manner in HeLa Cells and BMDM. To investigate if the changes in the CL:PI ratio were specific to the 

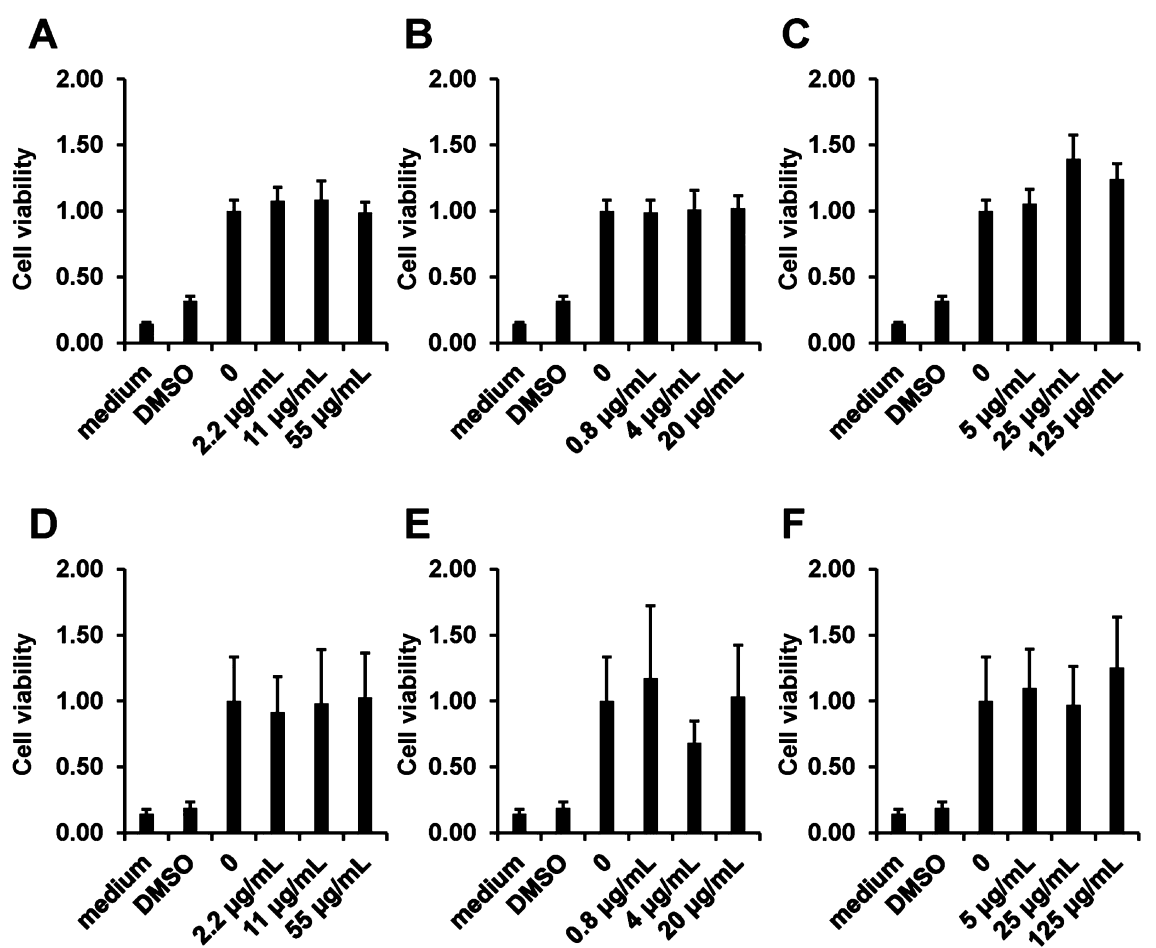

Figure 6. HeLa cells $(\mathrm{A}-\mathrm{C})$ and $\mathrm{BMDM}$ cells $(\mathrm{D}-\mathrm{F})$ viability expressed as a ratio of antibiotic-treated cells $\left({ }^{1} /{ }_{5} C_{\max }, C_{\max }\right.$ and $\left.5 C_{\max }\right)$ to untreated cells. Cells treated with levofloxacin (A and D), ethambutol (B and E), and kanamycin (C and F).
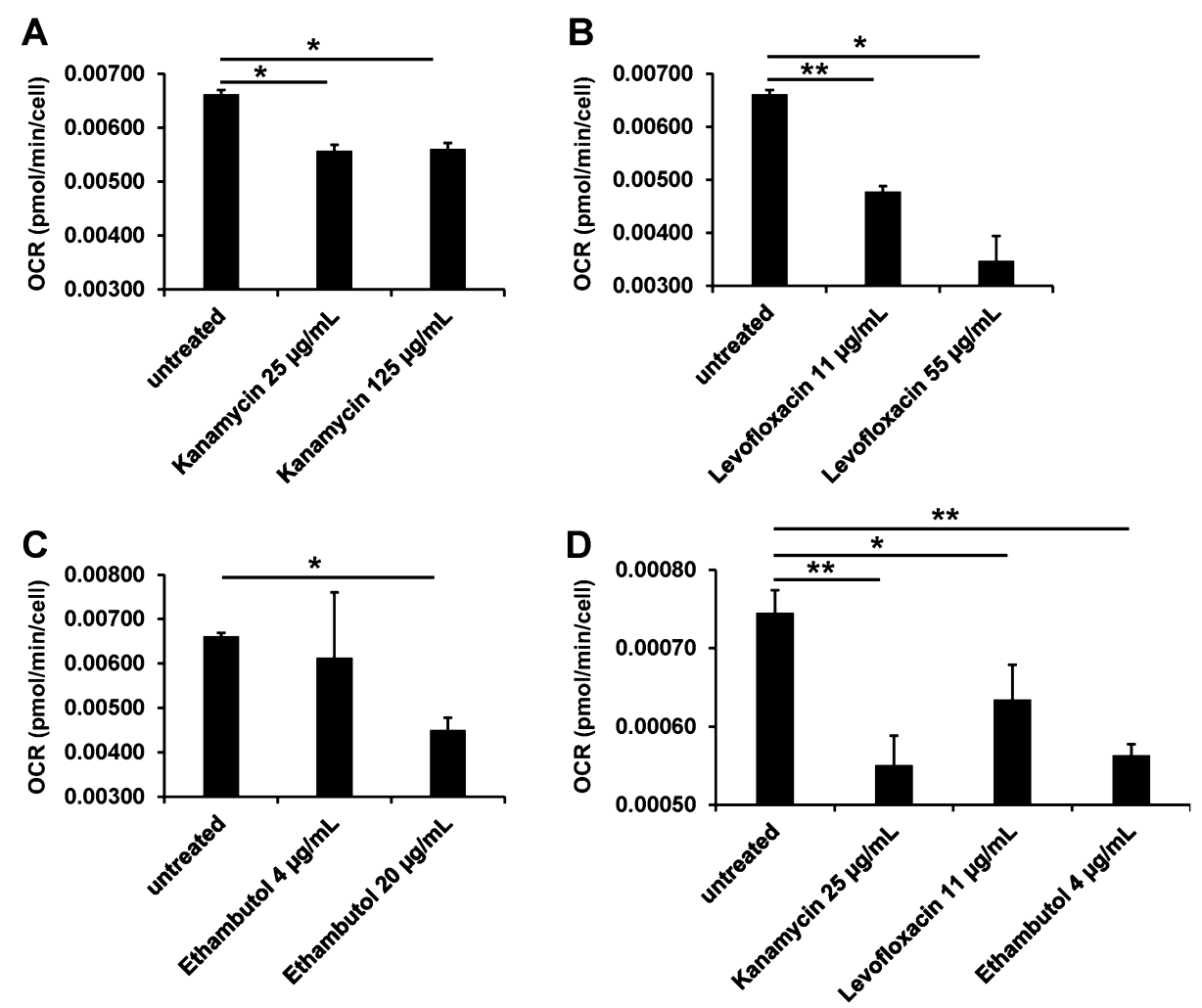

Figure 7. Maximal mitochondrial oxygen consumption in HeLa cells untreated and treated with kanamycin (A), levofloxacin (B), and ethambutol (C) and BMDM cells untreated and treated with kanamycin, levofloxacin, and ethambutol at $C_{\max }(\mathrm{D}) *^{*} p<0.05$ and $* * p<0.005$, as analyzed by a Student's $t$ test.

kanamycin exposure of HeLa cells for $24 \mathrm{~h}$ or to lipidome remodeling in response to antibiotic exposure, we conducted a series of ICL experiments by determining the CL:PI ratio of HeLa to BMDM cells after a $24 \mathrm{~h}$ exposure to levofloxacin, ethambutol, or kanamycin, each antibiotic having a different mode of action. We evaluated the CL:PI ratio after antibiotic treatment for $24 \mathrm{~h}$ using both HeLa and BMDM cells (primary cells), as they differ in their primary carbon metabolism. While 
immortalized cell lines such as HeLa cells catabolize glucose through the Embden-Meyerhof-Parnas pathway, the pentose phosphate pathway, and to a lesser extent the TCA cycle (Warburg effect), primary cells mainly employ the TCA cycle. $^{48-50}$ This can have major consequences for how the cells can respond to antibiotic stress exposure and so for the CL:PI ratio.

In both HeLa and BMDM cells, only $5 C_{\max }$ of levofloxacin ( 55 $\mu \mathrm{g} / \mathrm{mL}$ ) alters the CL:PI ratio with a 2.8 -fold decrease in HeLa cells and 1.5-fold decrease in BMDM cells (Figure 5). In addition, in both HeLa and BMDM cells, for all $C_{\max }$ values used, ethambutol exposures of $24 \mathrm{~h}$ do not alter the CL:PI ratio. However, in HeLa cells, kanamycin induces changes in the CL:PI ratio at $C_{\max }$ and $5 C_{\max }$ with similar orders of magnitude with an $\sim 1.5$-fold decrease in the CL:PI ratio. This effect is more pronounced in primary cells, where a dose dependence is noticed. In this case, at $C_{\max }$ the CL:PI ratio decreases by 1.4fold and by 2.5 -fold at $5 C_{\max }$. Taken together, discovered from use of ICL, these data suggest that only kanamycin at $C_{\max }$ induces changes in the CL:PI ratio.

Kanamycin, Levoflocaxin, and Ethambutol Do Not Affect HeLa Cells or BMDM Viability at $C_{\max }$ and $5 C_{\max }$. To determine if the changes in the CL:PI ratio were due to the difference in cell viability, we tested cell survival after $24 \mathrm{~h}$ exposure to the antibiotics. In this case, in terms of cell viability, no statistically significant differences were observed across all antibiotics and concentrations tested (Figure 6). These data confirm the hypothesis that changes in the CL:PI ratio are due to the antibiotic exposure and not due to cell apoptosis.

Kanamycin, Levoflocaxin, and Ethambutol Decrease the Maximal Rate of Mitochondrial Respiration. To test whether changes in the CL:PI ratio can directly correlate with mitochondrial dysfunction, we measured the maximal mitochondrial respiratory capacity on HeLa cells or BMDM cells treated with kanamycin, levofloxacin, and ethambutol at $1 /{ }_{5} C_{\max }$ $C_{\max }$ and $5 C_{\max }$ for HeLa cells and $C_{\max }$ for BMDM cells. We monitored changes in the oxygen consumption rate (OCR) of intact HeLa cells and BMDM using the Agilent Seahorse XF cell mito stress test. This assay measures directly the OCR and uses modulators of cellular respiration that specifically target components of the ETC to reveal key parameters of metabolic function. The compounds, oligomycin, FCCP, and a mix of rotenone and anitmycin A, are serially injected to measure ATP production, maximal respiration, and nonmitochondrial respiration, respectively. Oligomycin inhibits ATP synthetase (complex V), and FCCP is an uncoupling agent that collapses the proton gradient and disrupts the mitochondrial membrane potential. The OCR was measured in picomoles per minute and was normalized by the number of cells in each well. For HeLa cells, all antibiotics used led to a decrease in the maximal rate of mitochondrial respiration (Figure $7 \mathrm{~A}-\mathrm{C}$ ). Although the treatment of HeLa cells with kanamycin did not exhibit a dose dependence (Figure 7A), the treatment of HeLa cells with levofloxacin did exhibit a dose dependence (Figure 7B). With regards to ethambutol, only $5 C_{\max }$ led to a decrease in the maximal rate of mitochondrial respiration (Figure $7 \mathrm{C}$ ). For BMDM, at $C_{\max }$ all antibiotics used in this study led to a drastic decrease in the maximal mitochondrial respiratory capacity (Figure 7D).

Taken together, our data suggest that kanamycin, levofloxacin, and ethambutol can induce mitochondrial dysfunction. Our results are in accordance with results found in the literature. ${ }^{6,51,52}$ However, only kanamycin impairs the CL:PI ratio in a dose-dependent manner without affecting cell viability (Figures 2, 5, and 6). At this stage, from the use of ICL and determination of the CL:PI ratio, our results demonstrate that even if mitochondrial function was slightly impaired by the use of antibiotics such as ethambutol or levofloxacin at $C_{\max }$, there is no direct correlation between mitochondrial dysfunction and the CL:PI ratio. Only the aminoglycoside kanamycin can trigger changes in the CL:PI ratio. Effectively, our results based on the ICL and CL:PI ratio are in line with those found in the literature where aminoglycosides, including kanamycin, have been shown to modulate phosphoinositide signaling and metabolism in the cytoplasm and in the nucleus. ${ }^{53-58}$ We cannot rule out the possibility that a decrease in the maximal rate of mitochondrial oxygen consumption alter metabolic pathways; however, using state-of-art metabolomics tools such as high-sensitivity and highresolution liquid chromatography-mass spectrometry-based approaches will make this area exciting for future investigations.

\section{CONCLUSION}

Although the MALDI technique does not distinguish between lipid isobaric species, i.e., complex lipids with the same mass but different fatty acid composition, the purpose of this study was to provide a new approach for assessing antibiotic toxicity based on the lipid profile. By using MALDI-MS in the negative ion mode directly on intact and unprocessed HeLa cells and BMDM cells, we are able to perform targeted lipidomic profiling of PI and CL. This is achieved using as few as $100-1000$ cells per MALDI well with limited sample preparation. Thus, this method represents a major advance and can be used as an alternative to rapidly assess as a screening assay the toxicity of a compound that interferes with PI metabolism prior to in-depth analysis of the entire lipidome using conventional lipidomics approaches such as the ones presented earlier. We strongly believe that this methodology can go well beyond the scope of this study and can also be applied to more cell types and purposes such as lipidome remodeling to probe cancer diagnostics/metabolism and hostpathogen interactions.

\section{ASSOCIATED CONTENT}

\section{Supporting Information}

The Supporting Information is available free of charge on the ACS Publications website at DOI: 10.1021/acs.chemrestox. 8 b00038.

MS/MS spectra and data of PI and CL lipids (PDF)

\section{AUTHOR INFORMATION}

\section{Corresponding Author}

*E-mail: g.larrouy-maumus@imperial.ac.uk. Phone: +44 (0) 20 75947463.

\section{ORCID}

Gerald Larrouy-Maumus: 0000-0001-6614-8698

\section{Author Contributions}

All authors designed the study. S.R.-R., S.K., and G.L.-M. performed, analyzed, and interpreted the experiments.

\section{Funding}

This work was supported by an EPSRC-EMBRACE pumppriming award (EP/M027007/1). S.R.-R. is funded by the Department of Life Sciences from the Faculty of Natural Sciences of Imperial College London. Work in laboratory of S.M. is supported by a Wellcome Trust Senior Research Fellowship (206444/Z/17/Z), a Wellcome Trust Research 
Career Development Fellowship (WT097411MA), and the Lister Institute of Preventive Medicine.

\section{Notes}

The authors declare no competing financial interest.

\section{ACKNOWLEDGMENTS}

The authors acknowledge the mass spectrometry facility of the Department of Life Sciences at Imperial College London for providing access to the MALDI mass spectrometer. The authors also thank Dr. Alex Liversage and Dr. Nick Howe from Agilent Technologies for their helpful discussion and advice regarding the Seahorse data presented in this work.

\section{REFERENCES}

(1) Brummett, R. E., and Fox, K. E. (1989) Aminoglycoside-induced hearing loss in humans. Antimicrob. Agents Chemother. 33, 797-800.

(2) Mingeot-Leclercq, M. P., and Tulkens, P. M. (1999) Aminoglycosides: nephrotoxicity. Antimicrob. Agents Chemother. 43, 10031012.

(3) Kohanski, M. A., Dwyer, D. J., Hayete, B., Lawrence, C. A., and Collins, J. J. (2007) A common mechanism of cellular death induced by bactericidal antibiotics. Cell 130, 797-810.

(4) Dwyer, D. J., Kohanski, M. A., Hayete, B., and Collins, J. J. (2007) Gyrase inhibitors induce an oxidative damage cellular death pathway in Escherichia coli. Mol. Syst. Biol. 3, 91.

(5) Shatalin, K., Shatalina, E., Mironov, A., and Nudler, E. (2011) H2S: a universal defense against antibiotics in bacteria. Science 334, 986-990.

(6) Kalghatgi, S., Spina, C. S., Costello, J. C., Liesa, M., MoronesRamirez, J. R., Slomovic, S., Molina, A., Shirihai, O. S., and Collins, J. J. (2013) Bactericidal antibiotics induce mitochondrial dysfunction and oxidative damage in Mammalian cells. Sci. Transl. Med. 5, 192 ra85.

(7) Kohanski, M. A., Tharakan, A., Lane, A. P., and Ramanathan, M., Jr. (2016) Bactericidal antibiotics promote reactive oxygen species formation and inflammation in human sinonasal epithelial cells. Int. Forum Allergy Rhinol 6, 191-200.

(8) Tirmenstein, M. A., Hu, C. X., Gales, T. L., Maleeff, B. E., Narayanan, P. K., Kurali, E., Hart, T. K., Thomas, H. C., and Schwartz, L. W. (2002) Effects of troglitazone on HepG2 viability and mitochondrial function. Toxicol. Sci. 69, 131-138.

(9) Westwood, F. R., Bigley, A., Randall, K., Marsden, A. M., and Scott, R. C. (2005) Statin-induced muscle necrosis in the rat: distribution, development, and fibre selectivity. Toxicol. Pathol. 33, 246-257.

(10) Shah, R. R. (2006) Can pharmacogenetics help rescue drugs withdrawn from the market? Pharmacogenomics 7, 889-908.

(11) Siramshetty, V. B., Nickel, J., Omieczynski, C., Gohlke, B. O., Drwal, M. N., and Preissner, R. (2016) WITHDRAWN-a resource for withdrawn and discontinued drugs. Nucleic Acids Res. 44, D1080-1086.

(12) Belenky, P., Ye, J. D., Porter, C. B., Cohen, N. R., Lobritz, M. A., Ferrante, T., Jain, S., Korry, B. J., Schwarz, E. G., Walker, G. C., and Collins, J. J. (2015) Bactericidal Antibiotics Induce Toxic Metabolic Perturbations that Lead to Cellular Damage. Cell Rep. 13, 968-980.

(13) Zampieri, M., Zimmermann, M., Claassen, M., and Sauer, U. (2017) Nontargeted Metabolomics Reveals the Multilevel Response to Antibiotic Perturbations. Cell Rep. 19, 1214-1228.

(14) Paradies, G., Paradies, V., De Benedictis, V., Ruggiero, F. M., and Petrosillo, G. (2014) Functional role of cardiolipin in mitochondrial bioenergetics. Biochim. Biophys. Acta, Bioenerg. 1837, 408-417.

(15) Mejia, E. M., and Hatch, G. M. (2016) Mitochondrial phospholipids: role in mitochondrial function. J. Bioenerg. Biomembr. $48,99-112$.

(16) Layre, E., and Moody, D. B. (2013) Lipidomic profiling of model organisms and the world's major pathogens. Biochimie 95, 109-115.

(17) Sampaio, J. L., Gerl, M. J., Klose, C., Ejsing, C. S., Beug, H., Simons, K., and Shevchenko, A. (2011) Membrane lipidome of an epithelial cell line. Proc. Natl. Acad. Sci. U. S. A. 108, 1903-1907.
(18) Schuhmann, K., Herzog, R., Schwudke, D., Metelmann-Strupat, W., Bornstein, S. R., and Shevchenko, A. (2011) Bottom-up shotgun lipidomics by higher energy collisional dissociation on LTQ Orbitrap mass spectrometers. Anal. Chem. 83, 5480-5487.

(19) Lee, J. W., Mok, H. J., Lee, D. Y., Park, S. C., Kim, G. S., Lee, S. E., Lee, Y. S., Kim, K. P., and Kim, H. D. (2017) UPLC-QqQ/MS-Based Lipidomics Approach To Characterize Lipid Alterations in Inflammatory Macrophages. J. Proteome Res. 16, 1460-1469.

(20) Pinto, G., Caira, S., Mamone, G., Ferranti, P., Addeo, F., and Picariello, G. (2014) Fractionation of complex lipid mixtures by hydroxyapatite chromatography for lipidomic purposes. J. Chromatogr. A $1360,82-92$.

(21) Reis, A., Rudnitskaya, A., Blackburn, G. J., Mohd Fauzi, N., Pitt, A. R., and Spickett, C. M. (2013) A comparison of five lipid extraction solvent systems for lipidomic studies of human LDL. J. Lipid Res. 54, $1812-1824$.

(22) Dennis, E. A., Deems, R. A., Harkewicz, R., Quehenberger, O., Brown, H. A., Milne, S. B., Myers, D. S., Glass, C. K., Hardiman, G., Reichart, D., Merrill, A. H., Jr., Sullards, M. C., Wang, E., Murphy, R. C., Raetz, C. R., Garrett, T. A., Guan, Z., Ryan, A. C., Russell, D. W., McDonald, J. G., Thompson, B. M., Shaw, W. A., Sud, M., Zhao, Y., Gupta, S., Maurya, M. R., Fahy, E., and Subramaniam, S. (2010) A mouse macrophage lipidome. J. Biol. Chem. 285, 39976-39985.

(23) Lam, S. M., Tian, H., and Shui, G. (2017) Lipidomics, en route to accurate quantitation. Biochim. Biophys. Acta, Mol. Cell Biol. Lipids 1862, $752-761$.

(24) Angelini, R., Vitale, R., Patil, V. A., Cocco, T., Ludwig, B., Greenberg, M. L., and Corcelli, A. (2012) Lipidomics of intact mitochondria by MALDI-TOF/MS. J. Lipid Res. 53, 1417-1425.

(25) Angelini, R., Babudri, F., Lobasso, S., and Corcelli, A. (2010) MALDI-TOF/MS analysis of archaebacterial lipids in lyophilized membranes dry-mixed with 9-aminoacridine. J. Lipid Res. 51, 28182825.

(26) Tipthara, P., and Thongboonkerd, V. (2016) Differential human urinary lipid profiles using various lipid-extraction protocols: MALDITOF and LIFT-TOF/TOF analyses. Sci. Rep. 6, 33756.

(27) Alharbi, F. J., Geberhiwot, T., Hughes, D. A., and Ward, D. G. (2016) A Novel Rapid MALDI-TOF-MS-Based Method for Measuring Urinary Globotriaosylceramide in Fabry Patients. J. Am. Soc. Mass Spectrom. 27, 719-725.

(28) Lopalco, P., Angelini, R., Lobasso, S., Kocher, S., Thompson, M., Muller, V., and Corcelli, A. (2013) Adjusting membrane lipids under salt stress: the case of the moderate halophilic organism Halobacillus halophilus. Environ. Microbiol. 15, 1078-1087.

(29) Jones, J. J., Stump, M. J., Fleming, R. C., Lay, J. O., Jr., and Wilkins, C. L. (2004) Strategies and data analysis techniques for lipid and phospholipid chemistry elucidation by intact cell MALDI-FTMS. J. Am. Soc. Mass Spectrom. 15, 1665-1674.

(30) Larrouy-Maumus, G., Clements, A., Filloux, A., McCarthy, R. R., and Mostowy, S. (2016) Direct detection of lipid A on intact Gramnegative bacteria by MALDI-TOF mass spectrometry. J. Microbiol. Methods 120, 68-71.

(31) Larrouy-Maumus, G., and Puzo, G. (2015) Mycobacterial envelope lipids fingerprint from direct MALDI-TOF MS analysis of intact bacilli. Tuberculosis (Oxford, U. K.) 95, 75-85.

(32) Giannella, R. A., Washington, O., Gemski, P., and Formal, S. B. (1973) Invasion of HeLa cells by Salmonella typhimurium: a model for study of invasiveness of Salmonella. J. Infect. Dis. 128, 69-75.

(33) Mostowy, S., Sancho-Shimizu, V., Hamon, M. A., Simeone, R., Brosch, R., Johansen, T., and Cossart, P. (2011) p62 and NDP52 proteins target intracytosolic Shigella and Listeria to different autophagy pathways. J. Biol. Chem. 286, 26987-26995.

(34) Stavru, F., Bouillaud, F., Sartori, A., Ricquier, D., and Cossart, P. (2011) Listeria monocytogenes transiently alters mitochondrial dynamics during infection. Proc. Natl. Acad. Sci. U. S. A. 108, 36123617.

(35) Koberle, M., Klein-Gunther, A., Schutz, M., Fritz, M., Berchtold, S., Tolosa, E., Autenrieth, I. B., and Bohn, E. (2009) Yersinia enterocolitica targets cells of the innate and adaptive immune system 
by injection of Yops in a mouse infection model. PLoS Pathog. 5, e1000551.

(36) Kotra, L. P., Haddad, J., and Mobashery, S. (2000) Aminoglycosides: perspectives on mechanisms of action and resistance and strategies to counter resistance. Antimicrob. Agents Chemother. 44, $3249-3256$.

(37) von Baum, H., Bottcher, S., Abel, R., Gerner, H. J., and Sonntag, H. G. (2001) Tissue and serum concentrations of levofloxacin in orthopaedic patients. Int. J. Antimicrob. Agents 18, 335-340.

(38) Swoboda, S., Oberdorfer, K., Klee, F., Hoppe-Tichy, T., von Baum, H., and Geiss, H. K. (2003) Tissue and serum concentrations of levofloxacin $500 \mathrm{mg}$ administered intravenously or orally for antibiotic prophylaxis in biliary surgery. J. Antimicrob. Chemother. 51, 459-462.

(39) Drlica, K., and Zhao, X. (1997) DNA gyrase, topoisomerase IV, and the 4-quinolones. Microbiol. Mol. Biol. Rev. 61, 377-392.

(40) Babalik, A., Ulus, I. H., Bakirci, N., Kuyucu, T., Arpag, H., Dagyildiz, L., and Carpaner, E. (2013) Pharmacokinetics and serum concentrations of antimycobacterial drugs in adult Turkish patients. Int. J. Tuberc Lung Dis 17, 1442-1447.

(41) Imming, P., Sinning, C., and Meyer, A. (2006) Drugs, their targets and the nature and number of drug targets. Nat. Rev. Drug Discovery 5, 821-834.

(42) Urso, R., Blardi, P., and Giorgi, G. (2002) A short introduction to pharmacokinetics. Riv. Eur. Sci. Med. Farmacol. 6, 33-44.

(43) Holmes, D. T., and Buhr, K. A. (2007) Error propagation in calculated ratios. Clin. Biochem. 40, 728-734.

(44) Schiller, J., Suss, R., Fuchs, B., Muller, M., Petkovic, M., Zschornig, O., and Waschipky, H. (2007) The suitability of different DHB isomers as matrices for the MALDI-TOF MS analysis of phospholipids: which isomer for what purpose? Eur. Biophys. J. 36, 517-527.

(45) Amacher, D. E. (2005) Drug-associated mitochondrial toxicity and its detection. Curr. Med. Chem. 12, 1829-1839.

(46) Nadanaciva, S., and Will, Y. (2011) Investigating mitochondrial dysfunction to increase drug safety in the pharmaceutical industry. Curr. Drug Targets 12, 774-782.

(47) Shin, J. W., Park, S. H., Kang, Y. G., Wu, Y., Choi, H. J., and Shin, J. W. (2016) Changes, and the Relevance Thereof, in Mitochondrial Morphology during Differentiation into Endothelial Cells. PLoS One 11, e0161015.

(48) Liberti, M. V., and Locasale, J. W. (2016) The Warburg Effect: How Does it Benefit Cancer Cells? Trends Biochem. Sci. 41, 211-218.

(49) Kentner, D., Martano, G., Callon, M., Chiquet, P., Brodmann, M., Burton, O., Wahlander, A., Nanni, P., Delmotte, N., Grossmann, J., Limenitakis, J., Schlapbach, R., Kiefer, P., Vorholt, J. A., Hiller, S., and Bumann, D. (2014) Shigella reroutes host cell central metabolism to obtain high-flux nutrient supply for vigorous intracellular growth. Proc. Natl. Acad. Sci. U. S. A. 111, 9929-9934.

(50) Eisenreich, W., Heesemann, J., Rudel, T., and Goebel, W. (2013) Metabolic host responses to infection by intracellular bacterial pathogens. Front. Cell. Infect. Microbiol. 3, 24.

(51) Yu, M., Li, R., and Zhang, J. (2016) Repositioning of antibiotic levofloxacin as a mitochondrial biogenesis inhibitor to target breast cancer. Biochem. Biophys. Res. Commun. 471, 639-645.

(52) Guillet, V., Chevrollier, A., Cassereau, J., Letournel, F., Gueguen, N., Richard, L., Desquiret, V., Verny, C., Procaccio, V., Amati-Bonneau, P., Reynier, P., and Bonneau, D. (2010) Ethambutol-induced optic neuropathy linked to OPAl mutation and mitochondrial toxicity. Mitochondrion 10, 115-124.

(53) Marche, P., Koutouzov, S., and Girard, A. (1983) Impairment of membrane phosphoinositide metabolism by aminoglycoside antibiotics: streptomycin, amikacin, kanamycin, dibekacin, gentamicin and neomycin. J. Pharmacol. Exp. Ther. 227, 415-420.

(54) Marche, P., Olier, B., Girard, A., Fillastre, J. P., and Morin, J. P. (1987) Aminoglycoside-induced alterations of phosphoinositide metabolism. Kidney Int. 31, 59-64.

(55) Jiang, H., Sha, S. H., and Schacht, J. (2006) Kanamycin alters cytoplasmic and nuclear phosphoinositide signaling in the organ of Corti in vivo. J. Neurochem. 99, 269-276.
(56) Arbuzova, A., Martushova, K., Hangyas-Mihalyne, G., Morris, A. J., Ozaki, S., Prestwich, G. D., and McLaughlin, S. (2000) Fluorescently labeled neomycin as a probe of phosphatidylinositol-4, 5-bisphosphate in membranes. Biochim. Biophys. Acta, Biomembr. 1464, 35-48.

(57) De Matteis, M. A., Di Campli, A., and Godi, A. (2005) The role of the phosphoinositides at the Golgi complex. Biochim. Biophys. Acta, Mol. Cell Res. 1744, 396-405.

(58) Holz, R. W., Hlubek, M. D., Sorensen, S. D., Fisher, S. K., Balla, T., Ozaki, S., Prestwich, G. D., Stuenkel, E. L., and Bittner, M. A. (2000) A pleckstrin homology domain specific for phosphatidylinositol 4, 5bisphosphate (PtdIns-4,5-P2) and fused to green fluorescent protein identifies plasma membrane PtdIns-4,5-P2 as being important in exocytosis. J. Biol. Chem. 275, 17878-17885. 